\title{
A new method to measure bone properties by means of leaky surface acoustic waves
}

\author{
Noriyoshi Chubachi, Hitoshi Asai, and Toshio Sannomiya \\ Department of Electrical Engineering, Faculty of Engineering, Tohoku University, \\ Aramaki Aza-Aoba, Aoba-ku, Sendai, 980 Japan
}

(Received 17 September 1991)

\begin{abstract}
Experiments on excitation and detection of leaky surface acoustic waves on bones have been performed using linearly converging lens at a frequency of $1 \mathrm{MHz}$. In the experiments, leaky surface skimming compressional waves (LSSCWs) have been successfully excited on a polished flat surface of bovine bone with a coupling medium of water. Phase velocities of LSSCWs have been measured by the $V(z)$ curve analysis method developed in acoustic microscopy. The system is expected to develop a new type of diagnostic equipment for bones.
\end{abstract}

Keywords: Acoustic properties of bone, Acoustic microscopy, Velocity measurement, Surface acoustic wave, Leaky surface skimming compressional wave

PACS number: 43. 80. Vj, 43. 85. -e

\section{INTRODUCTION}

Measurements of bone properties by ultrasound have been attempted since the early $1950 \mathrm{~s}$. Basic characteristics of bones have been measured in vitro, and relations between acoustic properties and bone structure have been investigated by some researchers, such as Lang, ${ }^{1)}$ Yoon and Katz, ${ }^{2)}$ and Lakes. ${ }^{3)}$ In the medical field, the measurement of acoustic properties of bones is expected to be used to evaluate the state of fracture healing or to examine bone diseases such as osteoporosis. ${ }^{4,5)}$

Studies by some researchers to develop a practical diagnostic system for bone diseases have been in progress since 1980. Yoon and Katz proposed a system to measure the properties of femoral bones in vivo by acoustic emission techniques. ${ }^{6}$ ) Heaney, et al. carried out experiments to measure velocities of patella by longitudinal ultrasonic waves in vivo. ${ }^{7,8}$

Recently, we have proposed a new idea on the extracorporeal measurement system for bones by introducing leaky surface acoustic wave technology. It has been found that the leaky surface skimming compressional waves (LSSCWs) can be excited on bone samples with polished surfaces at low frequencies around $1 \mathrm{MHz}$. This paper describes the principle of the method to measure the bone properties through leaky surface acoustic waves and presents experimental results obtained with bovine bones.

\section{PRINCIPLE OF THE MEASUREMENT METHOD BY MEANS OF LEAKY SURFACE ACOUSTIC WAVES}

In acoustic microscopy, the properties of materials are determined by leaky surface acoustic waves excited on the samples. In this paper, we applied the same method developed in acoustic microscopy to measure the properties of bones. That is, the velocities of leaky surface acoustic waves on the sample are measured by the conventional $V(z)$ curve analysis described previously. ${ }^{9)}$

\section{$2.1 \quad V(z)$ Curve Analysis}

Figure 1 shows cross-sectional geometry of the ultrasonic sensor and sample coupled with water. Sensor outputs as a function of distance $z$ between lens and sample are called as $V(z)$ curves. A $V(z)$ 


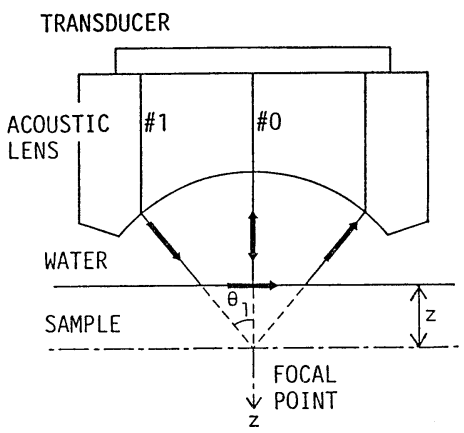

Fig. 1 Cross-sectional geometry of ultrasonic sensor and paths of two acoustic waves which contribute $V(z)$ curve construction.

curve has many periodical maxima and minima. The periodicity $\Delta z$ is related to the velocity of the leaky surface acoustic wave excited on the sample surface. So we can determine the velocity by analyzing the $V(z)$ curve as described below.

The periodicity $\Delta z$ on $V(z)$ curve are explained by the interference of two components of acoustic waves, $\# 0$ and $\# 1$, which propagate along the paths illustrated in Fig. 1. The component of $\# 0$ is associated with the waves near the $z$ axis and directly reflected from the sample surface. On the other hand, the component of $\# 1$ is associated with the waves reradiated from the sample into water through leaky surface acoustic waves excited on the watersample boundary. The relative phase difference between the components $\# 0$ and $\# 1$ varies with distance $z$, and a phase change of $2 \pi$ corresponds to the periodicity $\Delta z$, which is given by

$$
\Delta z=v_{\mathrm{w}} /\left\{2 f\left(1-\cos \theta_{\mathrm{i}}\right)\right\} \text {. }
$$

$\theta_{1}$, the critical angle to excite the leaky surface acoustic wave, is given by

$$
\theta_{1}=\sin ^{-1}\left(v_{\mathrm{w}} / v_{1}\right),
$$

where $v_{\mathrm{w}}$ is the longitudinal velocity in water, and $v_{1}$ is the leaky surface acoustic wave velocity, respectively.

Substituting Eq. (2) into Eq. (1), $v_{1}$ is given by Eq. (3) as a function of $\Delta z$.

$$
v_{1}=v_{\mathrm{w}} /\left[1-\left\{1-v_{\mathrm{w}} /(2 f \Delta z)\right\}^{2}\right]^{1 / 2}
$$

\subsection{Measurement System}

Figure 2 is a block diagram of the system for excitation and detection of leaky surface acoustic

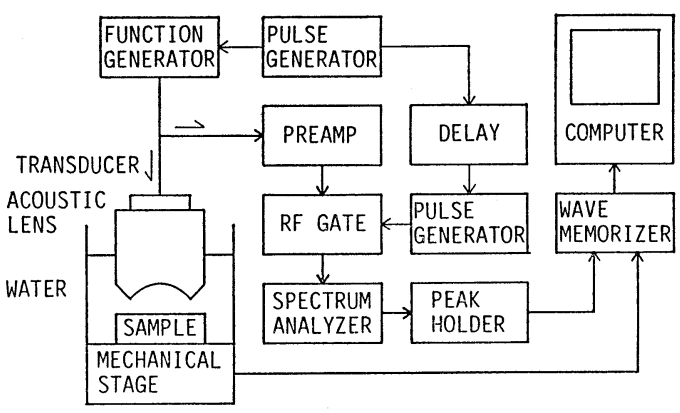

Fig. 2 Block diagram of the system for excitation and detection of leaky surface acoustic waves.

Table 1 Critical angles for excitation of leaky surface acoustic waves on bone surface.

\begin{tabular}{lrll}
\hline \multicolumn{2}{c}{ Velocity $[\mathrm{m} / \mathrm{s}]$} & \multicolumn{2}{c}{ Critical angle } \\
\hline$v_{x x}$ & 3,360 & $26^{\circ}$ & (LSSCW) \\
$v_{y y}$ & 3,410 & $26^{\circ}$ & (LSSCW) \\
$v_{z z}$ & 4,290 & $20^{\circ}$ & (LSSCW) \\
$v_{y z}$ & 2,013 & $48^{\circ}$ & (LSAW) \\
$v_{z x}$ & 1,873 & $53^{\circ}$ & (LSAW) \\
$v_{x y}$ & 1,736 & $59^{\circ}$ & (LSAW) \\
\hline
\end{tabular}

waves. The construction of this system is basically similar to the line-focus-beam acoustic microscope system. ${ }^{9)}$ In order to reduce propagation loss in the bone sample, the frequency of the system is much lower than that of conventional acoustic microscopes. In this preliminary study, a frequency of $1 \mathrm{MHz}$ is used. A piezoelectric ultrasonic sensor with cylindrical lens has been employed for excitation and detection of the leaky surface acoustic waves.

To design the lens, we have to take into account the critical angle at which leaky surface acoustic waves can be excited on the bone surface. In the estimation of the critical angles, we considered that velocities of leaky surface acoustic waves of Rayleigh-type (LSAWs) and leaky surface skimming compressional waves (LSSCWs) are nearly equal to those of shear and longitudinal wave velocities, respectively. Table 1 shows the critical angles estimated by Eq. (1) using the velocities of water $\left(1,483 \mathrm{~m} / \mathrm{s}\right.$ at $\left.20^{\circ} \mathrm{C}\right)$ and bones, where the orthorhomnic symmetry model given by $\mathrm{Katz}^{10)}$ is taken for the coordinate system in which the $z$ axis is 


\section{N. CHUBACHI et al.: A NEW METHOD TO MEASURE BONE PROPERTIES}

along the bone axis. In the table, longitudinal wave velocities $\left(v_{x x}, v_{y y}\right.$, and $\left.v_{z z}\right)$ are our measured data ${ }^{11}$ and shear wave velocities $\left(v_{y z}, v_{z x}\right.$, and $\left.v_{x y}\right)$ are estimated from the stiffness constants given by Kayz, ${ }^{10}$ ) providing the density of bovine bones is $2.0 \times 10^{3}$ $\mathrm{kg} / \mathrm{m}^{3}$. For LSSCW excitation, the angle should be less than $27^{\circ}$, while for LSAW excitation, the angle should be greater than $48^{\circ}$. The aperture half-angle of the lens must be larger than the critical angles for the leaky surface wave excitation. Considering the convenience of fabrication, we designed the lens with aperture half-angle of $40^{\circ}$ for the excitation of LSSCW mode. The LSSCW mode is considered to be preferable for in-vivo measurements because the propagation behavior of LSSCWs may be less affected by the surface roughness of bones compared to that of LSAWs.

Figure 3 shows a configuration of the ultrasonic sensor employed in this experiments. The lens material is aluminum (velocity; $6,300 \mathrm{~m} / \mathrm{s}$ ) and a PZT plate (center frequency; $1 \mathrm{MHz}$ ) is bonded as a transducer. Aperture half-angle is $40^{\circ}$ and the radius of curvature of the lens is $55 \mathrm{~mm}$ in order to obtain $V(z)$ curves with enough distance $z$ to observe many periodical dips for analysis. A vinylchloride sheet of a quarter wavelength thick (density; $1.2 \times 10^{3} \mathrm{~kg} / \mathrm{m}^{3}$, velocity; $2,400 \mathrm{~m} / \mathrm{s}$ ) is attached to the aperture as an acoustic matching layer. The side wall of the aluminum lens has been roughed and painted with absorbing material of tungsten and epoxy resin composite to suppress the spurious noise in the lens. The thickness of the absorbing layer is a few millimeters.

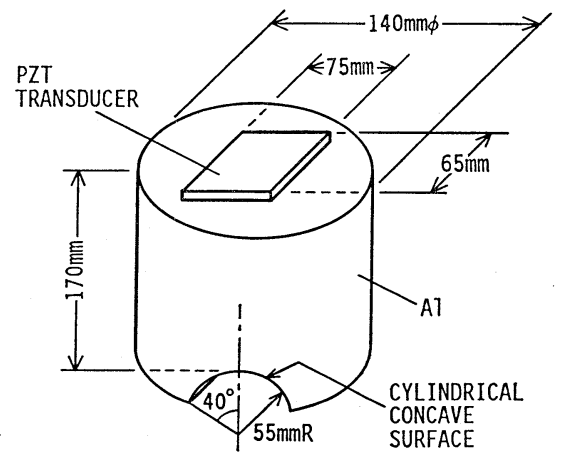

Fig. 3 Ultrasonic sensor with cylindrical lens for excitation and detection of leaky surface acoustic waves.

\section{EXPERIMENTS}

\subsection{System Performance}

To evaluate and check the system performance, we have measured $V(z)$ curves for Pyrex and Bakelite plates. Sample thicknesses are $20 \mathrm{~mm}$ and $10 \mathrm{~mm}$, respectively. Figures 4 (a) and (b) show the $V(z)$ curves measured at $1 \mathrm{MHz}$ for Pyrex and Bakelite, respectively. Clear dips can be seen in the figures and the dip intervals are evaluated to be $6.31 \mathrm{~mm}$ for Pyrex and $8.28 \mathrm{~mm}$ for Bakelite. Substituting these values to Eq. (3), the velocities for Pyrex and

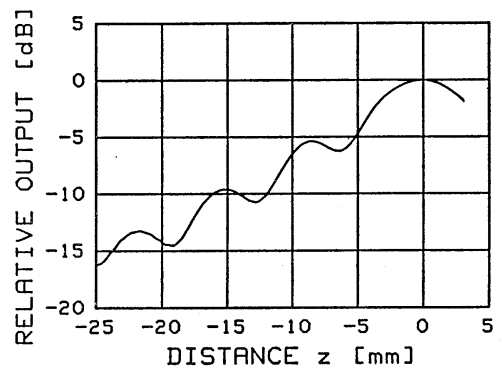

(a) Pyrex

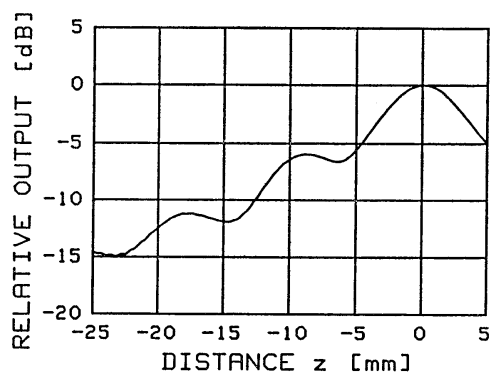

(b) Bakelite

Fig. $4 \quad V(z)$ curves obtained for Pyrex and Bakelite plates.

Table 2 Measured leaky wave velocities of Pyrex and Bakelite.

\begin{tabular}{lccccc}
\hline & Measured & & \multicolumn{3}{c}{ Literature } \\
\cline { 2 - 3 } \cline { 5 - 6 } & $\begin{array}{c}\text { Leaky } \\
\text { wave }\end{array}$ & $\begin{array}{c}\text { Longi- } \\
\text { tudinal } \\
\text { wave }\end{array}$ & $\begin{array}{c}\text { Shear } \\
\text { wave }\end{array}$ & $\begin{array}{c}\text { LSAW } \\
(225 \\
\text { MHz) }\end{array}$ \\
\hline Pyrex & $\begin{array}{c}3,153 \\
(\text { LSAW) } \\
\text { Bakelite }\end{array}$ & $5,560^{12)}$ & $3,430^{12)}$ & $3,132^{12)}$ \\
$\left(\begin{array}{l}3,585 \\
\text { (LSCW) }\end{array}\right.$ & $3,676^{11)}$ & - & - \\
\hline
\end{tabular}


Bakelite are determined to be $3,153 \mathrm{~m} / \mathrm{s}$ and 3,585 $\mathrm{m} / \mathrm{s}$, respectively. These values are listed in Table 2, with bulk wave velocities and a LSAW velocity quoted from the literatures ${ }^{11,12}$ ) for comparison. Comparing the leaky wave velocities with bulk wave velocities, it is seen that leaky waves excited in Pyrex are LSAW and that in Bakelite are LSSCW. According to these results, the system can be evaluated to perform sufficiently enough to investigate bone whose leaky wave velocity is close to that of Bakelite.

\subsection{Measurement of Bone Properties}

In the measurement of bone properties, bovine femoral bone was taken as a sample and its surface was polished by $20 \mu \mathrm{m}$-lapping paper. The sample was cut from whole bone and is $30 \mathrm{~mm}$ long, $40 \mathrm{~mm}$ wide, and $3-5 \mathrm{~mm}$ thick as shown in Fig. 5. $V(z)$ curve measurements were made for the sample immersed in water. Figure 6 is the $V(z)$ curve obtained for a propagating direction along to the bone axis. Dips of this curve are not so clear as those observed for Bakelite. It may be because of higher attenuation caused by scattering and/or viscosity of bone. The phase velocity is determined to be 3,924 $\mathrm{m} / \mathrm{s}$, the $\Delta z$ being estimated to be $10.0 \mathrm{~mm}$. The

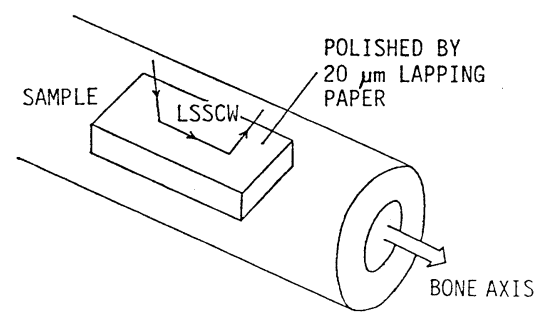

Fig. 5 Sample preparation with respect to bone axis.

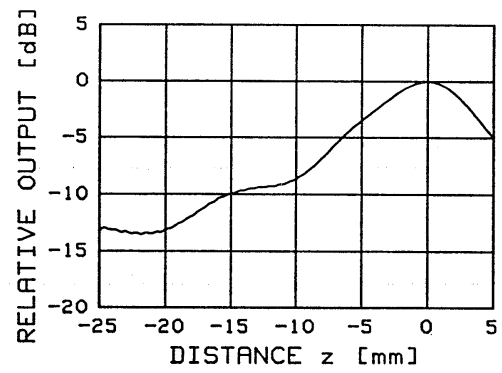

Fig. $6 V(z)$ curve obtained for polished bone surface. value of this phase velocity is rather close to the longitudinal bulk wave velocity $v_{z z}$ listed in Table 1 , so that the mode of leaky surface waves excited on the bone may be confirmed as a LSSCW mode. Considering the penetration depth of LSSCW is about a wave length, it can be expected by the measurements at $1 \mathrm{MHz}$ that bone properties from surface to a depth of $4 \mathrm{~mm}$ will be reflected in the measured values.

\section{CONCLUSION}

In order to develop new diagnostic equipment for evaluating bones by ultrasound, a system based on acoustic microscopy technique has been investigated. A measurement system with a linearly converging lens sensor operated at a frequency of $1 \mathrm{MHz}$ has been constructed to obtain $V(z)$ curves on bone samples in vitro. It has been found that the system is capable of exciting and detecting LSSCW in the bone sample.

To realize an in-vivo system, this system can be modified for application to the human body. Fortunately, in the in-vivo measurements of bones, soft tissues such as skin or muscle surrounding the bones can be used as a part of the coupling medium together with coupling water because velocities of soft tissues are close to that of water. Most importantly, the configuration of the transducers should be redesigned according to the shape of the bones to be examined.

It is expected that the development of this measurement method will result in practical diagnostic equipment for bones which will be clinically employed.

\section{ACKNOWLEDGEMENTS}

The authors are grateful to $\mathrm{S}$. Hisa and $\mathrm{N}$. Ichikawa for their kind assistance in this work.

\section{REFERENCES}

1) S. B. Lang, "Ultrasonic method for measuring elastic coefficients of bone and results on fresh and dried bovine bones," IEEE Trans. Biomed. Eng. BME-17, 101-105 (1970).

2) H. S. Yoon and J. L. Katz, "Ultrasonic wave propagation in human cortical bone II, Measurements of elastic properties and microhardness," J. Biomech. 9, 459-464 (1976).

3) R. Lakes, H. S. Yoon, and J. L. Katz, "Ultrasonic wave propagation and attenuation in wet bone," J. Biomed. Eng. 8, 143-148 (1986). 


\section{N. CHUBACHI et al.: A NEW METHOD TO MEASURE BONE PROPERTIES}

4) I. M. Siegel, G. T. Anast, and T. Field, "The determination of fracture healing by measurement of sound velocity across the fracture site," Surg. Gynecol. Obstet. 107, 327-332 (1958).

5) J. D. Craven, M. A. Constantini, M. A. Greenfield, and R. Stern, "Measurement of the velocity of ultrasound in human cortical bone and its potential clinical importance. An in vivo preliminary study," Invest. Radiol. 8, 72-77 (1973).

6) H. S. Yoon, B. Caraco, H. Kaur and J. L. Katz, "Clinical application of acoustic emission techniques to bone abnormalities," 1980 IEEE Ultrason. Symp. Proc. Cat. No. 80CH1602-2 2, 1067-1072 (1980).

7) R. P. Heaney, L. V. Avioli, C. H. Chesnut III, J. Lappe, R. R. Recker, and G. H. Brandenburger, "Osteoporotic bone fragility detection by ultrasound transmission velocity," JAMA 261, 29862990 (1989).

8) G. H. Brandenburger, L. V. Avioli, C. H. Chesnut III, R. P. Heaney, R. Poss, G. Pratt, and R. R. Recker, "In-vivo measurement of osteoporotic bone fragillity with apparent velocity of ultrasound," 1989 IEEE Ultrason. Symp. Proc. Cat. No. 89CH2791-2 2, 1023-1027 (1989).

9) J. Kushibiki and N. Chubachi, "Material characterization by line-focus-beam acoustic microscope," IEEE Trans. Sonics Ultrason. SU-32, 189-212 (1985).

10) J. L. Katz, "The biophysical and biomechanical properties of bone, bone mineral and some synthetic bone biomaterials," Bul. Soc. Chem. Fr. 1985, 514-518 (1985).

11) N. Chubachi, T. Sannomiya, and H. Asai, "Measurement of bone properties by ultrasound to develop diagnostic equipment," 1990 IEEE Ultrason. Symp. Proc. Cat. No. 90CH2938-9 3, 1367$1370(1990)$.

12) J. Kushibiki, T. Ueda, and N. Chubachi, "Determination of elastic constants by LFB acoustic microscope,' 1987 IEEE Ultrason. Symp. Proc. Cat. No. 87CH2492-7 2, 817-821 (1987).

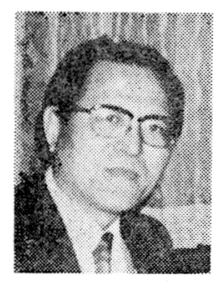

Noriyoshi Chubachi was born in Fukuoka Prefecture, Japan, on October 5, 1933. He received the B.S., M.S., and Ph.D. degrees in electrical engineering from Tohoku University, Sendai, Japan, in 1956, 1962, and 1965, respectively. In 1965 he joined the Research Institute of Electrical
Communication, Tohoku University, where he was an Associate Professor from 1966 to 1978. Since 1979 he has been a Professor at the Department of Electrical Engineering, Tohoku University. Since 1974 Dr. Chubachi has been working to develop a new scientific field of ultrasonic micro-spectroscopy including acoustic microscopy. He is a member of many institutes including the IEEE, the IEICE of Japan, the Japan Society of Applied Physics and the Japan Society of Ultrasonics in Medicine. He served as chairman of the Tokyo chapter of the IEEE UFFC Society from 1987 to 1988 , and of the Tohoku chapter of the Acoustical Society of Japan from 1988 to 1990 . He is currently chairman of the Tohoku chapter of the Japan Society of Applied Physics.

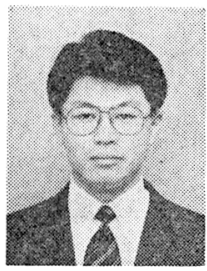

Hitoshi Asai was born in Miyagi Prefecture, Japan, on November 20, 1963. He graduated from the Department of Electronic Engineering, Yamagata University, Yonezawa, Japan, in 1986. He received the degree of M.S. in electrical engineering from Tohoku University, Sendai, Japan, in 1988. In 1990 he became a Research Associate at the Department of Electrical Engineering, Faculty of Engineering, Tohoku University. He has been interested in ultrasonic microspectroscopy, especially field of biological tissue characterization. Mr. Asai is a member of the Japan Society of Applied Physics and the Japan Society of Ultrasonics in Medicine. He received the Awaya Kiyoshi Award in 1991.

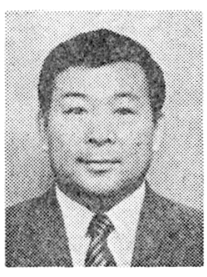

Toshio Sannomiya was born in Miyagi Prefecture, Japan, on October 3, 1938. He graduated from the Sendai Radio High School in 1959. From 1959 to 1978 , he was with the Research Institute of Electrical Communication, Tohoku University, Sendai, Japan. Since 1979, he has been working at the Department of Electrical Engineering, Faculty of Engineering, Tohoku University. He has engaged in the studies of ultrasonic transducers, ultrasonic measurements, and acoustic microscopy. $\mathrm{Mr}$. Sannomiya is a member of the Japan Society of Ultrasonics in Medicine. 